\title{
Perceived Shape at a Slant as a Function of Processing Time and Processing Load
}

\author{
William Epstein, Gary Hatfield, and Gerard Muise \\ University of Wisconsin-Madison
}

\begin{abstract}
Shape and slant judgments of rotated or frontoparallel ellipses were elicited from three groups of 10 subjects. A masking stimulus was introduced to control processing time. Backward masking trials were presented with interstimulus intervals of 0,25 , and $50 \mathrm{msec}$. Reduction of processing time altered shape judgments in the direction of projective shape and slant judgments in the direction of frontoparallelness. This finding is consistent with the shapeslant invariance hypothesis. In order to study the effects of processing load, one group of subjects was given prior knowledge of the kind of judgment to be made on each trial, one group had no prior knowledge, and a third group made both judgments on each trial. The effects of the processing load manipulation were interpreted in terms of the role of attention in perceptual encoding. Consistent with previous findings, allocation of attention did not affect perceptual encoding.
\end{abstract}

Leibowitz and Bourne (1956) found that exposure duration affected the shape judgment of a circle rotated in depth. Reduction of exposure duration was accompanied by deviations from shape constancy, shape matches that were in closer agreement with projective shape than with objective shape. For exposure durations of $100 \mathrm{msec}$ and less, the matches conformed perfectly to projective shape, that is, the subject selected as a match an elliptical shape equal to the frontoparallel projection of the rotated circle.

The effect of exposure duration is analogous to the effect of reduction of depth information in a variety of perceptual constancy experiments (Epstein, 1973, 1977; Epstein \& Park, 1963; Epstein, Park, \& Casey, 1961). In fact, Leibowitz and Bourne (1956) suggested that the effect of exposure duration on shape judgment may have been mediated by perceived orientation. The shape-slant invariance hypothesis (Epstein, 1973; Epstein \& Park, 1963) pro-

Requests for reprints should be sent to William Epstein, Department of Psychology, University of Wisconsin, Madison, Wisconsin 53706. vides a context for elaboration of Leibowitz and Bourne's speculation. According to this hypothesis, projective shape determines an invariant relationship between perceived shape and perceived slant. Since only a single objective shape-objective orientation combination was used in the Leibowitz and Bourne experiment, projective shape was the same on all trials. Consequently, to be consistent with the shape-slant hypothesis, the reductions of exposure duration should have been accompanied by underestimations of perceived slant. For the $100-\mathrm{msec}$ exposure, which yielded a perfect projective shape match, the circle should have been perceived as frontoparallel, although objectively it was rotated $30^{\circ}$. One aim of the present experiment was to evaluate this interpretation by securing slant judgments as well as shape judgments.

The procedure we employed differed in three essential respects from Leibowitz and Bourne's (1956) procedure. (a) Rather than presenting a single standard shape repeatedly at a single orientation, we presented two families of shape-slant combinations. Within each family, all shape-slant combinations produced the same projective shape. This arrangement allowed a more satisfac- 
tory evaluation of the effect of exposure duration on perceived shape at a slant by testing discrimination of differences in objective shape in the presence of projective equivalence. (b) In four unreported experiments in which we followed Leibowitz and Bourne's procedure of varying exposure time, we consistently found significant effects of exposure time: At very brief durations, judged shape tended toward projective shape; while for longer durations, for example, $500 \mathrm{msec}$, constancy was nearly perfect. Although these findings were in general agreement with Leibowitz and Bourne, we were unable to secure perfect projective shape matches even with an exposure duration as low as $10 \mathrm{msec}$. Our lack of success in this respect led us to question the adequacy of the exposure duration manipulation. A simple manipulation of exposure time provides no control of processing in the period immediately following stimulus offset. For this reason, exposure time and processing time probably are not synchronous. Accordingly, in the present study, instead of varying exposure time we varied processing time. Exposure time was constant on all trials for an individual subject; processing time was varied by varying the stimulus onset asynchrony in a backward masking paradigm. (c) In Leibowitz and Bourne's procedure, all of the shapes that constituted the comparison series from which subjects selected the match to the standard had the same vertical-linear dimension as the standard. Consequently, the possibility cannot be dismissed that subjects based their responses on a match between the projected horizontal extents rather than shape match. In our comparison series, the shapes were scale reductions, so that subjects were induced to make shape judgments.

The second aim of our study was to determine whether perception of shape at a slant is affected by attentional control and temporal capacity limitations. Our concern may be explained by comparing Leibowitz and Bourne's (1956) procedure with the present procedure. In the former case, the subjects were tested only for shape; while in the present case, both shape and slant perception were tested. Consider the first procedure. Are we justified in drawing conclusions about perceiving shape at a slant when a subject has been directed to report only shape? It may be contended that since shape and slant normally are packaged as a single perceptual unit, emphasis on only one of these elements alters the task of perceptual processing. Despite the fact that questions of this sort often are adumbrated in the literature on perceptual constancies, there has been no direct empirical investigation of the matter.

The question was evaluated in the present study by varying the specificity and timing of the tests. On only tests, the subject was informed in advance that only a single attribute, shape or slant, would be tested. On both tests, the subject was informed in advance that both shape and slant would be tested. On either tests, the subject was informed that either shape or slant would be probed, but that the attribute would be designated after presentation of the standard. We looked at two aspects of the performance under these three conditions: (a) the effect on shape judgments and slant judgments and (b) the effect on the relationship between perceived shape and perceived slant. If testing a single property, shape or slant, alters the nature of the process, then performance should not be the same on only and both tests. The either tests were equivalent to both tests in respect to processing load and equivalent to only tests in terms of demands on retrieval. In the event that a difference between only and both tests is obtained, the either tests should help to determine whether the difference is in initial processing or in retrieval. The rationale is reminiscent of the logic underlying the analysis of selective attention in the experiments of Massaro (1975), Shiffrin, Gardner, and Allmeyer (1973), and Shiffrin, McKay, and Shaffer (1976).

The variations of test type also bear on a methodological problem in experimental assessments of the various algorithms for space perception (Epstein, 1973), for example, the size-distance invariance hypothesis and the shape-slant invariance hypothe- 
sis. When the algorithms are assessed by examining the correlations between two perceptual variables, for example, perceived size and perceived distance or perceived shape and perceived slant, in what sequence should the to-be-correlated judgments be secured? When the judgments of the same test object are secured in immediate succession, first one and then the other, there is the risk of inflating the correlation due to induction of a response set that favors packaging the two judgments in a reasonable way. In order to avoid this potential artifact, the judgments of the same object often are separated by intervening judgments of other targets. However, this procedure entails the risk that the intervening experience has modified the perceptual or response system, so that the obtained correlation does not accurately reflect the relationship that prevailed at either the occasion of the first probe or the second. Examination of the correlations between perceived shape and perceived slant across the three trial types in the present experiment will show whether these methodological concerns are in fact warranted.

\section{Method \\ Subjects \\ Thirty-four undergraduates participated in the experiment for course credit, money $(\$ 2.50$ per session), or a combination of the two at their option.}

\section{Stimuli}

The standard stimuli were eight ellipses cut from $1 / 16$ in. $(.159 \mathrm{~cm})$ white posterboard and affixed to $2 \frac{1}{2}$ in. $(6.35 \mathrm{~cm})$ high black stalks. The edges of the stimuli were beveled to minimize edge cues. The length of the vertical axis in each standard was $5.3 \mathrm{~cm}$. The horizontal axes varied from 3.29 to $15.64 \mathrm{~cm}$. The stimuli were partitioned into two sets, Set 1 consisting of four stimuli having horizontal-vertical axis ratios of $.62, .79,1.00$, and 1.46; and Set 2 consisting of four stimuli having horizontal-vertical axis ratios of 1.25, 1.61, 2.03, and 2.95. Within each set, one shape was presented at only one orientation, $0^{\circ}$ (frontoparallel), $39^{\circ}$, $52^{\circ}$, or $65^{\circ}$. For half of the subjects, the stimuli in Set 1 were rotated clockwise, and those in Set 2 were rotated counterclockwise; for the other half, the converse obtained. The objective shapeobjective orientation pairs were such that all shapes in Set 1 projected a horizontal-vertical ratio of .62 (vertical subtense of $4.5^{\circ}$, horizontal subtense of $2.8^{\circ}$ ), and all shapes in Set 2 projected a horizontal-vertical ratio of 1.25 (vertical subtense of $4.5^{\circ}$, horizontal subtense of $\left.5.5^{\circ}\right) .1$ The masking stimulus consisted of randomly shaped and randomly arranged pieces of white paper, each less than 1 square centimeter in size, attached to black matte construction paper.

The comparison stimuli for shape were 11 scaled-down drawings, each with a vertical axis of $2.2 \mathrm{~cm}$. The horizontal axes varied to produce shapes with horizontal-vertical axis ratios ranging from .45 to 3.8 . The shapes were arranged on single sheets of paper in a single column, either in ascending or descending order. Embedded within the 11 comparison shapes were the 8 shapes corresponding to the horizontal-vertical axis ratios of the stimuli, one shape narrower than any of the stimuli (ratio of .45), one wider (ratio of 3.8 ), and two that served as interpolations between the shapes of the first and second stimuli in each set (ratios of .70 and 1.46, the latter acting both as an intermediate and as a match to Set 1).

The comparison stimuli for orientation were 13 circles $(2.2 \mathrm{~cm}$ in diameter), each containing a single line drawn along a diameter. Each drawing represented a top view of the stimulus at one orientation relative to the observer. The represented orientations ranged from $78^{\circ}$ counterclockwise through 0 through $78^{\circ}$ clockwise in $13^{\circ}$ increments. The stimuli were arranged on a single sheet of paper in either ascending or descending order.

Iindividual sheets of comparison stimuli were prepared for each trial and placed in a predetermined random order in a loose-leaf binder, so that only the current trial information was available to the subject. Each comparison stimulus was numbered, and the subject recorded the response by writing its number on a new index card for each trial.

\section{Apparatus}

The apparatus was a modified two-field tachistoscope controlled by an Automated Data System 1248 timer. Each arm of the tachistoscope was 54 $\mathrm{cm}$ long, $23.5 \mathrm{~cm}$ wide, and $25 \mathrm{~cm}$ high. A lightproof extension of the direct-view channel of the tachistoscope provided access to a calibrated disk into which the stimuli could be inserted. The masking stimulus was in the reflected arm of the tachistoscope at a distance of $59 \mathrm{~cm}$ from the viewer's eyes. The standard stimuli were located $67 \mathrm{~cm}$

1 The shapes within each set were equivalent when projected orthogonally onto the frontoparallel plane. The projection of the shapes onto the retina was subject to perspective distortions (for rotated stimuli) and binocular disparity, so that the retinal projections of the shapes within each set were not precisely equivalent. 
Table 1

Mean Shape Judgment Averaged for all Eight Standards as a Function of Masking Condition and Test Type

\begin{tabular}{lcccc}
\hline & \multicolumn{4}{c}{ Masking condition } \\
\cline { 2 - 5 } & \multicolumn{3}{c}{$\begin{array}{c}\text { Interstimulus interval } \\
\text { (in msec) }\end{array}$} \\
\cline { 2 - 5 } \multicolumn{1}{c}{ Group } & 0 & 25 & 50 & No mask \\
\hline Only & 1.174 & 1.233 & 1.278 & 1.522 \\
Either & 1.298 & 1.363 & 1.396 & 1.547 \\
Both (shape first) & 1.242 & 1.268 & 1.303 & 1.411 \\
Both (slant first) & 1.246 & 1.280 & 1.306 & 1.475 \\
\hline
\end{tabular}

from the subject and were viewed binocularly through a $10 \times 4 \mathrm{~cm}$ window framed by a viewing hood that excluded ambient light and restricted head movement. The viewing field was restricted to the standard and the masking stimulus by occluders appropriately placed in each channel of the tachistoscope. Illumination was provided by a fluorescent light in each arm, located in front of and below the stimuli. The luminance level in each display field was $3.0 \mathrm{~cd} / \mathrm{m}^{2}$.

\section{Exposure Durations}

On all trials calling for a mask, the mask was exposed for $400 \mathrm{msec}$. The interstimulus interval was 0,25 , or $50 \mathrm{msec}$. When no mask was presented, offset of the standard was followed by an empty dark field. Exposure durations for the standards were determined for each subject individually in the manner explained below.

\section{Procedure}

A subject served in three 1-hour-long sessions, each separated by 24 hours. The instructions emphasized phenomenal (apparent) shape and orientation judgments (Carlson \& Tassone, 1967; Epstein, 1963; Epstein, Bontrager, \& Park, 1962). The subject was directed to base the response on an immediate impression of shape or orientation. The first session was devoted to determining the appropriate exposure duration for each subject individually. We searched for an exposure duration that satisfied two criteria: (a) approximation of the minimum duration that allowed a high degree of constancy (defined as a Brunswik ratio ${ }^{2}$ of .70 or higher) and (b) a duration that would be compatible with a significant masking effect (defined as a mask-induced reduction of the Brunswik ratio of at least .20 from the no-mask level). These criteria could not be satisfied for all subjects. Three subjects were dismissed for failure to exhibit a Brunswik ratio of at least .70 , and one was dismissed for failure to exhibit a reliable masking effect.

The following routine was used to establish the desired exposure duration. First there were eight trials, one for each standard, at a 500-msec exposure with no mask. Responses to both shape and orientation were elicited on each of these trials. A Brunswik ratio was calculated for the six standards that were rotated from the frontal plane. Another series of eight trials at $200 \mathrm{msec}$ with no mask followed, and another Brunswik ratio was calculated. These trials familiarized the subject with the procedure and the class of stimuli and helped the experimenter narrow the range of $\mathrm{du}$ rations to be considered. Two practice trials with the mask, followed by three blocks of 16 trials (eight mask and eight no-mask trials randomly interspersed) were then administered. The exposure duration for the first block was selected on the basis of the performance on the previous trials at 500 and $200 \mathrm{msec}$. A duration of $150 \mathrm{msec}$ was most often chosen. The interstimulus interval (ISI) on mask trials was always zero. Shape judgments were elicited on all of the trials and orientation judgments on three trials in each block of 16 to ensure that the subject would not adopt a shape-anly set. At the end of each block, a Brunswik ratio was calculated, and the next stimulus duration was adjusted recursively to converge to the above-mentioned criteria. Whenever ambiguity arose about which stimulus duration to use, the lower exposure duration was selected.

At the conclusion of the first session, the subjects were randomly assigned to three groups of 10 according to the type of test they would receive on the second and third days. The mean exposure durations for the three groups did not differ ( 70 , 87 , and $82 \mathrm{msec}$ for only, both, and either, respectively). The only group had to report either shape or slant on each trial and knew beforehand whether it was to be shape or slant. The either group also made only one judgment per trial but did not know until stimulation had been terminated whether shape or slant would be tested. The both group judged both shape and slant on each trial but were not informed beforehand which they were to report first.

Eight practice trials were presented on each day, followed by 64 test trials. For the only and either groups, the test trials on each day consisted of the eight standards presented at each of the three ISIs and with no mask, once for shape judgment and once for slant judgment. The both group received the eight standards at the four masking conditions presented once on each day for judgment of shape and then slant, once for judgment of slant and then shape. All subjects received different permutations of the test trials for all sessions.

2 A Brunswik ratio (Hochberg, 1971) is computed according to the formula $(a-p) /(o-p)$, where $a$ is judged or perceived shape, $p$ is projective shape, and $o$ is objective shape (shape is defined in terms of the width-height ratio). 

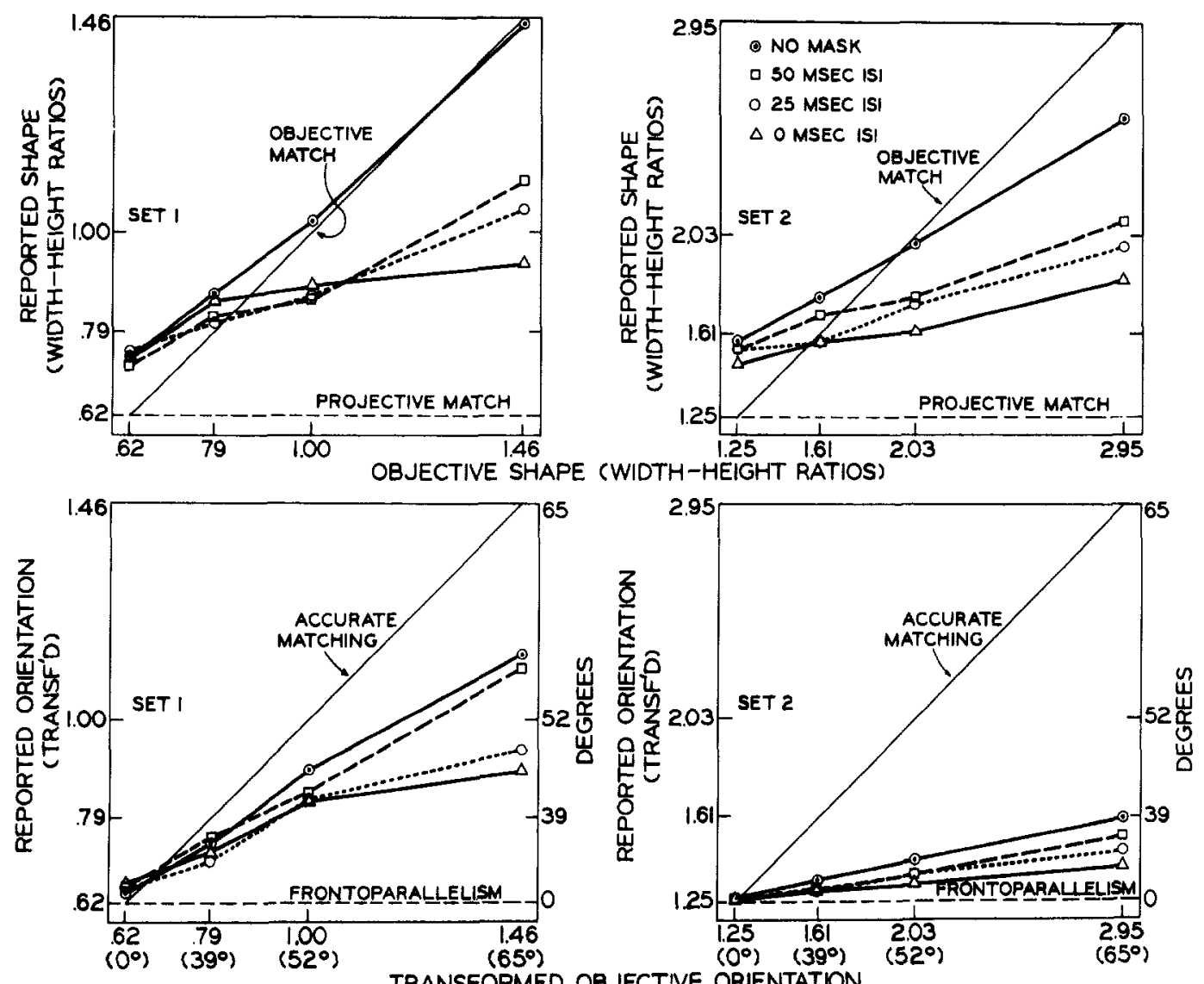

Figure 1. Mean shape scores and transformed mean orientation scores under each masking condition for all subjects across test type. (Sets 1 and 2 are projectively equivalent families of shapes. ISI $=$ interstimulus interval.)

\section{Results}

Separate analyses of variance (Test Type $\times$ Stimulus Set $\times$ Standard $\times$ Masking Condition) of shape and orientation judgments yielded no significant main effect or interaction of test type. The conclusion that test type did not influence the processing of shape at a slant is confirmed by the mean shape matches (expressed as width-height ratios) presented in Table 1 . Since test type was not a significant variable, the results presented henceforth pertain to mean scores across test type.

\section{Shape Judgments}

The upper half of Figure 1 shows the effect of the masking variable on judged shape. In the absence of a mask, the four shapes within each set were discriminated. The line labeled objective match and having a slope of 1.0 represents the set of judgments that would exhibit perfect constancy. The slopes of the best-fitting line for the judgments in the absence of the mask were .80 and .79 for Sets 1 and 2, respectively. The Brunswik ratio provides another summary of these results. A ratio of 1.0 represents perfect constancy. The obtained ratios in the absence of the mask were 1.03 and .90 for Sets 1 and 2, respectively.

It is also plain from Figure 1 that introduction of the mask drastically reduced constancy (the masking variable was significant at $p<.001)$. For the ISI of zero, the Bruns- 
Table 2

Standard Deviation of Shape Judgments for Each Stimulus Under Each Masking Condition

\begin{tabular}{|c|c|c|c|c|c|}
\hline \multirow{3}{*}{$\begin{array}{l}\text { Width-height ratio } \\
\text { of standard }\end{array}$} & \multirow{3}{*}{$\begin{array}{l}\text { Orientation } \\
\text { of standard }\end{array}$} & \multicolumn{4}{|c|}{ Masking condition } \\
\hline & & \multicolumn{3}{|c|}{ Interstimulus interval (in msec) } & \multirow[b]{2}{*}{ No mask } \\
\hline & & 0 & 25 & 50 & \\
\hline \multicolumn{6}{|c|}{ Set 1} \\
\hline $\begin{array}{r}.62 \\
.79 \\
1.00 \\
1.46\end{array}$ & $\begin{array}{r}0^{\circ} \\
39^{\circ} \\
52^{\circ} \\
65^{\circ}\end{array}$ & $\begin{array}{l}.190 \\
.404 \\
.289 \\
.296\end{array}$ & $\begin{array}{l}.209 \\
.252 \\
.219 \\
.400\end{array}$ & $\begin{array}{l}.152 \\
.187 \\
.226 \\
.362\end{array}$ & $\begin{array}{l}.195 \\
.203 \\
.282 \\
.575\end{array}$ \\
\hline \multicolumn{6}{|c|}{ Set 2} \\
\hline $\begin{array}{l}1.25 \\
1.61 \\
2.03 \\
2.95\end{array}$ & $\begin{array}{r}0^{\circ} \\
39^{\circ} \\
52^{\circ} \\
65^{\circ}\end{array}$ & $\begin{array}{r}.223 \\
.258 \\
.364 \\
.440\end{array}$ & $\begin{array}{l}.283 \\
.279 \\
.414 \\
.555\end{array}$ & $\begin{array}{l}.216 \\
.404 \\
.424 \\
.525\end{array}$ & $\begin{array}{l}.321 \\
.372 \\
.480 \\
.572\end{array}$ \\
\hline
\end{tabular}

wik ratios for Sets 1 and 2 were reduced to .58 and .46 , respectively. The slopes for this condition were .19 and .29 , respectively. A slope of zero would result from projective matching. The obtained slopes for the ISI of zero indicate that subjects only minimally discriminated among the projectively equivalent rotated and frontoparallel shapes within Sets 1 and 2. Figure 1 suggests that the effectiveness of the mask tended to diminish for longer ISIs, although even the ISI of 50 msec had a pronounced effect. The mask affected the judgment of rotated shapes only, which is reflected in a significant $(p<.001)$ Masking $\times$ Shape Withn Set interaction. Since for frontoparallel standards, objective and projective axis ratios do not differ, no masking effect was expected.

Examination of the $S D$ s reported in Table 2 shows a clear tendency for the variability of the shape judgments of the rotated shapes to be greater in the no-mask condition than in the masking conditions. Variability also increased in most conditions as the standards increased in width and degree of rotation, as can be seen by examination of the columns in Table 2 .

\section{Orientation Judgments}

Table 3 shows the mean orientation judgments. Without exception the shapes in Set
2 were judged to be less rotated than the shapes in Set 1 (the effect of set was significant, $p<.001)$. With the exception of the frontoparallel orientation, the orientations for Set 2 were greatly underestimated. As a rule, orientation judgments of the rotated shapes when the mask was present were smaller than when the mask was absent (the masking variable was significant, $p<.001)$. The slopes of the best-fitting lines shown in Table 3 increase regularly from ISIs of zero through the longer ISIs and are steepest when no mask was present, as is consistent with the significant $(p<$ .001) Masking $\times$ Slant Within Set interaction. Variabilities were not affected by masking or orientation.

In the lower half of Figure 1, the orientation judgments have been transformed so that the orientation and shape judgments are represented in a common metric. The transformed orientation values (TO) result from a nonlinear rescaling of the mean orientation values across subjects. The numerical value of a transformed score represents the shape (width-height ratio) that would be compatible with the actual projective shape of the standard involved and the obtained judged orientation in degrees. Thus, for the standard having a horizontal-vertical ratio of .62 and presented in the frontal 
plane, the transformation is as follows: $T O$ $=.62 / \cos \Theta$, where .62 is the projective shape of the standard, and $\Theta$ is the obtained orientation score in degrees. As orientation increases from $0^{\circ}$, the denominator in the above formula diminishes at a positively accelerated rate. Consequently, the transformation has the effect of compressing the scale for orientation between $0^{\circ}$ and about $35^{\circ}$, so that incremental changes in orientation within this range produce relatively small changes in $T O$.

Examination of the upper and lower halves of Figure 1 permits comparison of the effects of the mask on shape judgments with the effects on orientation judgments. Introduction of the mask and decreases in ISI moved the shape judgments away from objective matching. Consistent with the shape-slant invariance hypothesis, these deviations from shape constancy were accompanied by increasing underestimations of orientation as processing time was reduced. However, as is apparent from Figure 1, the effects of the mask on shape and orientation judgments were not precisely equivalent. In particular, the masking conditions produced a greater spread among the shape scores than among the slant scores. The relevance of these findings for the invariance hypothesis will be considered in the Discussion section.

Since exposure duration of the standard was determined independently for each subject, the durations (stimulus onset asynchronies, in the case of the masking trials) varied considerably among subjects. To determine the effect of this methodologically introduced variable, the subjects were grouped into five classes according to the following exposure durations: $25,50,75$, $100-125$, and $150-200 \mathrm{msec}$. Analysis of variance for unequal $N$ s using Henderson's Method I failed to yield a significant main effect or interaction of exposure duration for either shape or orientation judgments. Other features of this analysis were consistent with the findings reported earlier.

Table 3

Mean Orientation Scores in Degrees for Each Standard Under Each Masking Condition

\begin{tabular}{|c|c|c|c|c|}
\hline \multirow{3}{*}{$\begin{array}{l}\text { Orientation } \\
\text { of standard }\end{array}$} & \multicolumn{4}{|c|}{ Masking condition } \\
\hline & \multicolumn{3}{|c|}{ Interstimulus interval (in msec) } & \multirow[b]{2}{*}{ No mask } \\
\hline & 0 & 25 & 50 & \\
\hline \multicolumn{5}{|c|}{ Set 1} \\
\hline $\begin{array}{r}0 \\
39 \\
52 \\
65\end{array}$ & $\begin{array}{l}19.2(25.3) \\
30.7(22.3) \\
41.0(23.3) \\
45.5(23.4)\end{array}$ & $\begin{array}{l}17.5(24.12) \\
28.3(23.1) \\
41.5(20.4) \\
48.3(22.3)\end{array}$ & $\begin{array}{l}17.7(22.9) \\
34.4(24.1) \\
42.5(20.8) \\
55.5(17.4)\end{array}$ & $\begin{array}{l}13.4(20.0) \\
33.4(20.3) \\
45.7(18.8) \\
56.3(17.2)\end{array}$ \\
\hline \multicolumn{5}{|l|}{ Regression ${ }^{\mathrm{a}}$} \\
\hline $\begin{array}{l}\text { Slope } \\
\text { Intercept }\end{array}$ & 18.3 & 15.6 & $\begin{array}{l}.55 \\
11.8\end{array}$ & 11.97 \\
\hline \multicolumn{5}{|c|}{ Set 2} \\
\hline $\begin{array}{r}0 \\
39 \\
52 \\
65\end{array}$ & $\begin{array}{r}3.4(10.7) \\
14.3(16.6) \\
19.3(13.8) \\
25.8(16.1)\end{array}$ & $\begin{array}{r}3.3(6.7) \\
14.2(13.4) \\
22.9(16.0) \\
30.9(15.8)\end{array}$ & $\begin{array}{rr}3.2 & (9.7) \\
16.0 & (12.7) \\
23.7 & (13.2) \\
34.6 & (13.0)\end{array}$ & $\begin{array}{ll}1.5 & (4.0) \\
21.6 & (14.3) \\
28.3 & (12.69) \\
38.5 & (14.2)\end{array}$ \\
\hline $\begin{array}{l}\text { Regression }^{\mathrm{a}} \\
\text { Slope } \\
\text { Intercept }\end{array}$ & 2.73 & $\begin{array}{l}.41 \\
1.8\end{array}$ & -1.3 & $\begin{array}{l}.55 \\
.8\end{array}$ \\
\hline
\end{tabular}

Note. First entry is the mean; second entry (shown in parentheses) is the standard deviation.

a The regression analysis was performed for judged slant on objective slant. 
Table 4

Regression ( $\mathrm{r}$ ) Analysis of Judged Shape (Y) and Transformed Judged Slant (X) Based on Group Averages

\begin{tabular}{|c|c|c|c|c|}
\hline \multirow[b]{3}{*}{ Statistic } & \multicolumn{4}{|c|}{ Masking condition } \\
\hline & \multicolumn{3}{|c|}{ Interstimulus interval (in msec) } & \multirow[b]{2}{*}{ No mask } \\
\hline & 0 & 25 & 50 & \\
\hline \multicolumn{5}{|c|}{ Set 1} \\
\hline $\begin{array}{l}r_{x y} \\
Y \\
X\end{array}$ & $\begin{array}{c}.934 \\
.75 X+.27 \\
1.17 Y-.22\end{array}$ & $\begin{array}{c}.955 \\
.95 X+.13 \\
.96 Y-.06\end{array}$ & $\begin{array}{c}.995 \\
.84 X+.17 \\
1.18 Y-.19\end{array}$ & $\begin{array}{c}.976 \\
.76 X+.29 \\
1.25 Y-.32\end{array}$ \\
\hline \multicolumn{5}{|c|}{ Set 2} \\
\hline $\begin{array}{l}r_{x y} \\
Y \\
X\end{array}$ & $\begin{array}{c}.974 \\
3.60 X-.31 \\
.26 Y+.87\end{array}$ & $\begin{array}{c}.996 \\
2.19 X-.12 \\
.45 Y+.57\end{array}$ & $\begin{array}{c}.995 \\
1.97 X-.90 \\
.50 Y+.47\end{array}$ & $\begin{array}{c}.998 \\
2.80 X-.20 \\
.36 Y+.70\end{array}$ \\
\hline
\end{tabular}

\section{Correlation Between Judged Shape and} Judged Orientation

Table 4 shows that the correlations between judged shape and transformed orientation were high. This indicates that the function relating judged shape to judged orientation was nearly linear, as is predicted by the shape-slant invariance hypothesis. Since the transformed orientation scores are in the same metric as the shape scores, perfect congruence between orientation and shape judgments would result in a regression line slope of 1.0 . This would occur if the masking conditions had precisely equivalent effects upon shape and orientation judgments. Inspection of Table 4 reveals that this was much more nearly the case for judgments of the standards in Set 1 than for Set 2. The extreme slopes in Set 2 reflect the previously mentioned fact that the masking conditions produced a greater spread among shape scores than among slant scores. Nonetheless, from inspection of Figure 1, it is apparent that for both sets, if shape scores are adjusted downwards, taking the scores obtained for the frontoparallel standards (the standard with shape .62 in Set 1 and 1.25 in Set 2) as a base for projective shape matching, judged shape and judged slant become nearly congruent for the three masking conditions, while slant judgments still fall below shape judgments for the no-mask condition.

In order to further assess the effects of test type, a regression analysis was performed on the mean judged shape and transformed judged orientation scores within each instructional group. Comparison of the coefficients and regression line slopes revealed no consistent differences among the test types. However, an isolated exception bears mentioning. For Set 2 at the 0 - and 25-msec ISIs, the subjects who judged both shape and slant on the same trial exhibited higher correlation coefficients (range: .80 to .98; mean: .92) than those who judged shape and slant on separate trials (range: .07 to .98 ; mean : .48 ). Furthermore, the fact that the regression line slopes for the both group for Set 2, $0 \mathrm{msec}$ and $25 \mathrm{msec}$, were closer to 1.00 (range of deviation from $1.00: .09$ to .43 ) than the slopes for the other groups (range of deviation from 1.00: .45 to .93 ) indicates that the effects of the mask on shape and slant judgments were more nearly equivalent for the both group than for the other groups under these conditions. These isolated findings provide limited justification for the methodological concern, expressed in the introduction, that elicitation of both types of judgment on a single trial may influence the results in favor of the invariance hypothesis (high correla- 
tion and more nearly congruent shape and slant scores).

\section{Discussion}

The masking stimulus was utilized to control processing time. Introduction of the mask did not disrupt formation of the sensory icon, that is, contour formation, since for all of the ISIs the reports were within the range of values expected given intact projective shape information. Nor did the mask increase variability. In fact, as was noted, variability of the shape judgments was decreased by the introduction of the mask. The principal effect of the mask on perceived shape was to induce deviations from shape constancy, so that the inferred function relating judged shape to the objective shape of the standards was in better agreement with projective than with objective shape matching. The greatest effect was obtained with the ISI of zero. The 25- and 50-msec ISIs yielded shape judgments intermediate between the near projective match of the zero ISI and the near objective match of the no-mask condition.

In the introduction, we derived an interpretation of Leibowitz and Bourne's (1956) data from the general hypothesis that perceived shape is the result of combining projective shape information and perceived orientation. This account was intended to apply also to the effect of masking on perceived shape. Two expectations were generated by this account: (a) Introduction of a mask should lead to underestimation of orientation relative to judged orientation in the absence of a mask. (b) The effect of the mask on perceived orientation should be proportional to the effect on perceived shape. The obtained effect of the mask on judged orientation and the high correlations between shape and slant judgments satisfy both of these expectations. We conclude that the effect upon judged shape of the mask in our experiment and of exposure duration in Leibowitz and Bourne's study was mediated by an reffect upon the discrimination of orientation, in accordance with the shape-slant invariance hypothesis.
The two expectations just mentioned are consistent with a weak form of the shapeslant invariance hypothesis, which requires only that the effects of the mask on shape and slant perception be proportional. A stronger form of the invariance hypothesis would require perceived shape and perceived orientation to be in strict congruence with the shape-slant algorithm. For our data, this requirement would be satisfied only if the lines representing judged shape and transformed judged orientation in Figure 1 were congruent under each masking condition. While our data does not support the stronger form of the hypothesis, neither does it provide falsifying evidence. For our data to be sufficient as falsifying evidence, it would have to be assumed that reported or judged orientation is an accurate measure of orientation information as registered by the perceptual system and as combined with shape information to yield perceived shape. Since a distinction between registered and reported stimulus features has been proposed for other constancies, such as for size (Epstein, 1973, 1977, Chap. 13), the above assumption is unwarranted. Our data is therefore mute with respect to the strong form of the invariance hypothesis.

Early studies of shape constancy summarized by Koffka (1935, pp. 233-235) reported that constancy decreases as the degree of rotation from the frontal plane increases. This finding has been replicated by subsequent investigations (summarized by Epstein \& Park, 1963) and is buttressed by the observation of a similar falling off for size constancy with increasing viewing distances. However, reexamination of the results for the no-mask condition depicted in the top panels of Figure 1 shows that, in the present experiment, shape constancy varied little for rotations from frontoparallel up to $65^{\circ}$.

The discrepancy between the present and earlier results may be due to differences between our stimuli and those used previously: We varied objective shape as a function of orientation, keeping projective shape constant; whereas in the earlier studies, objective shape was held constant at all 
orientations. Since under our procedure object width increased with orientation, disparity and convergence cues were not degraded at extreme orientations as they would have been under the earlier procedure. Orientation was therefore less likely to be underestimated at extreme orientations, which is consistent with our finding that under the full-cue (no-mask) condition, the underestimation of orientation was proportionally equivalent at all orientations. The conventional explanation of the earlier finding that shape constancy diminishes as orientation increases attributes the diminution to poorer discrimination of extreme orientations, specifically to underestimation that increases as objective orientation increases. If the earlier results did reflect an underestimation of orientation, a comparison with our results suggests that it was because orientation cues were degraded at extreme orientations.

The earlier practice of holding objective shape constant at all orientations has another consequence that bears on the finding of underconstancy. As degree of stimulus rotation increases, variations of constant magnitude in orientation or projective shape are associated with relatively larger and larger changes in objective shape. This fact coupled with the shape-slant invariance algorithm has specific consequences for perceived shape. Strict application of the algorithm predicts that for a given objective shape, a constant deviation from the veridical in the registration of either orientation or projective shape will have a greater effect at more extreme orientations. Thus, if objective shape is held constant as degree of rotation increases, a slight under-registration of either orientation or projective shape will have a greater effect on perceived shape (by affecting perceived width for horizontally rotated stimuli) at larger orientations than at smaller ones. Consistent with the earlier findings, a larger degree of underconstancy is predicted at greater orientations than smaller ones. This consideration does not apply with our procedure. For our stimuli, objective width was larger for more extremely rotated stimuli, so that the effects of small perturbations in the registra- tion of shape and slant information would have a relatively small impact upon perceived shape at any orientation when compared to objective stimulus dimensions.

Variability of the shape judgments for rotated standards increased as inferred processing time increased and also as objective orientation increased. In the process underlying the perception of shape at a slant, which is summarized by the shape-slant invariance hypothesis, there are three possible loci of variability: (a) discrimination of orientation, (b) discrimination of projective shape, and (c) the stage that combines orientation and projective shape. The requisite pattern of variability was not obtained for orientation judgments nor for the frontoparallel stimuli (which provide a gauge for projective shape); therefore, the combinatorial stage is implicated. One account of the pattern of variability attributes it to cognitive factors present during the combinatorial stage. When the mask occurs immediately after onset of the standard, the combinatorial process may occur spontaneously without any accompanying cognitive adjustments. However, as processing time is increased, the opportunity for cognitive compensation for rotation increases. The likelihood of such cognitive intervention is expected to be greater at more extreme perceived rotations. The pattern of variability in the shape judgments may reflect differences in this hypothesized cognitive involvement. When the ISI is zero, there is no cognitive involvement and perceived shape is specified by the shape-slant invariance algorithm. Variability is low. At longer ISIs and especially at larger perceived rotations, individual differences in cognitive compensation lead to higher variability of reported shape. Although we have no systematic observations to bring to bear on this thesis, unsolicited remarks by our subjects were consistent with the thesis, particularly with respect to the effect of orientation on the intrusion of cognitive considerations.

Another account of the obtained pattern of variability follows from consideration of the shape-slant algorithm. As was discussed above, the algorithm predicts that variations in either orientation or projective shape 
have a greater effect upon perceived shape at extreme rotations than at more moderate rotations. For example, for our stimulus Set 1 , a deviation in registered orientation of $13^{\circ}$ leads to a deviation in perceived shape of $7.2 \%$ (expressed as percent of change in width-height ratio) when this deviation is computed for a stimulus with a mean registered orientation of $26^{\circ}$. However, if the mean registered orientation is $52^{\circ}$, a deviation of the same magnitude produces a $21 \%$ deviation in perceived shape. Thus, as registered orientation increases, the effect upon perceived shape of a constant error in registered orientation increases with positive acceleration. Moreover, since our stimuli were wider at greater orientations, the predicted effect of constant variability in projective shape is numerically larger variability in perceived shape at larger values of stimulus rotation. In conclusion, given that the effect upon perceived shape of a constant error in registered shape or orientation increases as registered orientation increases, the pattern of variability in the present results is predicted by straightforward application of the algorithm.

Variations in the allocation of attention presumably were associated with the different test types. Yet, test type had no consistent effect on perceived shape and orientation. In line with the claims of Shiffrin et al. (1973, 1976), it would appear that voluntary attentional control does not affect the encoding stage of visual processing. Our concern, expressed earlier, that testing only one of the attributes, for example, shape or slant, may alter the nature of the underlying process was not substantiated. It seems that whatever the emphasis of the test, the percipient always processes a shape at a slant. Probably for the same reason our results showed no consistent differences associated with the arrangement of tests in the degree of perceived shape-perceived orientation correlation: The measured relationship was the same when orientation and shape were tested separately on different trials as when orientation and shape were tested successively on the same trial, save for the exception mentioned in the Results section.
In assessing the significance of these findings for the claim that all attentional effects are postperceptual, it is worth noting that the possibility of selective allocation of attention could depend on the degree to which the to-be-attended and the to-be-unattended features normally are treated separately in perception. Since shape and orientation typically are packaged as a perceptual unit, our failure to show an effect of directed attention ought not to be generalized for cases that do not present the same degree of integration.

\section{References}

Carlson, V. R., \& Tassone, E. P. Independent size judgments at different distances. Journal of $E_{x}$ perimental Psychology, 1967, 73, 491-497.

Epstein, W. Attitudes of judgment and the sizedistance invariance hypothesis. Journal of $E_{x}$ perimental Psychology, 1963, 66, 78-83.

Epstein, W. The process of "taking-into-account" in visual perception. Perception, 1973, 2, 267-285.

Epstein, W. (Ed.). Stability and constancy in visual perception: Mechanisms and processes. Wiley-Interscience: New York, 1977.

Epstein, W., Bontrager, H., \& Park, J. The induction of nonveridical slant and the perception of shape. Journal of Experimental Psychology, $1962,63,472-479$.

Epstein, W., \& Park, J. Shape constancy: Functional relationships and theoretical formulations. Psychological Bulletin, 1963, 60, 265-288.

Epstein, W., Park, J., \& Casey, A. The current status of the size-distance hypothesis. Psychological Bulletin, 1961, 58, 491-514.

Hochberg, J. Perception: II. Space and movement. In J. W. Kling \& L. A. Riggs (Eds.), Woodworth and Schlosberg's experimental psychology. New York: Holt, Rinehart \& Winston, 1971.

Koffka, K. Principles of Gestalt psychology. New York: Harcourt Brace, 1935.

Leibowitz, H., \& Bourne, L. Time and intensity as determiners of perceived shape. Journal of Experimental Psychology, 1956, 51, 277-281.

Massaro, D. W. Backward recognition masking. Journal of the Acoustical Society of America, $1975,58,1059-1065$.

Shiffrin, R. M., Gardner, G. T., \& Allmeyer, D. H. On the degree of attention and capacity limitations in visual processing. Perception \& Psychophysics, 1973, 14, 231-236.

Shiffrin, R. M., McKay, D. P., \& Shaffer, W. O. Attending to forty-nine spatial positions at once. Journal of Experimental Psychology: Human Perception and Performance, 1976, 2, 14-22.

Received November 2, 1976 